\title{
SURFACE MODIFICATION OF NANOFIBROUS MEMBRANES FOR WATER PURIFICATION
}

\author{
${ }^{1}$ Izabela Joanna GALLUS, ${ }^{1}$ Evren BOYRAZ, ${ }^{1}$ Fatma YALCINKAYA \\ ${ }^{1}$ TUL - Technical University of Liberec, Faculty of Mechatronics, Informatics and Interdisciplinary Studies, \\ Liberec, Czech Republic, EU, izabela.gallus@tul.cz
}

https://doi.org/10.37904/nanocon.2021.4328

\begin{abstract}
Around 1.2 billion people have limited access to drinkable water and millions succumb every year to sicknesses caused due to the consumption of impure water or lack of water. In the coming years, this problem will only progress at alarming rates due to the aggressive growth of population, urbanization and droughts. It is essential to investigate new approaches for water treatment with low energy costs and economic benefits. The biggest problem in membrane filtrations is fouling, which causes membrane pore blockages and creates a cake layer on the membrane surface. The aim of the project is to obtain nanofibrous membranes that will have high fouling resistance and antibacterial effects. Nanoparticles of $\mathrm{Ag}, \mathrm{ZnO}, \mathrm{TiO} 2$ and $\mathrm{CuO}$ are known for their antibacterial properties. In order to attach nanoparticles to the membrane surface, appropriate functional groups are required. The first step of the project involves the attachment of functional groups capable of binding nanoparticles to the surface of the nanofiber membrane. For this alkaline surface modification using $\mathrm{NaOH}$, $\mathrm{KOH}, \mathrm{DETA}$ under different conditions was performed. After modification, changes in hydrophilicity and structure of membranes, pore size and appearance of nanofibers were checked. Defluorination reaction conditions with $\mathrm{NaOH}$ and $\mathrm{KOH}$ proved to be insufficient to obtain the desired functional groups on the membrane surface. In the case of the reaction with DETA, amine groups were obtained, which will be used in the next step to attach the nanoparticles.
\end{abstract}

Keywords: PVDF, nanofibers, surface, alkaline, modification

\section{INTRODUCTION}

Various methods have been already investigated in the purification of water like distillation, sand filtration, reverse osmosis, membrane filtration. Membrane filtration is a relatively new approach and has numerous advantages when compared to other methods. Nanofiltration allows the removal of minerals, salts, pathogens (bacteria, viruses, fungus), total dissolved solids (TDS), cations and anions from the water, requiring low power and low operational temperatures [1]. Till date, numerous nanomaterials have been developed for water treatment. Nanomaterials can be divided into various groups based on their physicochemical properties and surface characteristics. The most specified materials included carbon nanotubes (CNTs), nanofibers, polymerbased nanoadsorbents, nanoclays (natural and modified), nanoparticles ( $\mathrm{Au}, \mathrm{Ag}, \mathrm{Pt}$ NPs) and metal-based nanoadsorbents ( $\mathrm{Fe} 2 \mathrm{O} 3, \mathrm{ZnO}, \mathrm{CuO}, \mathrm{TiO} 2 \mathrm{NPs}$ ) and mixed oxide nanoparticles. Each of these materials have specific properties and should be used accordingly in the purification of, e.g., carbon nanotubes are recommended for removal organic contaminations, whereas bioactive nanoparticles are applied to remove fungi and bacteria [2]. Thin-Film Nanocomposite (TFC) membranes are prepared by interfacial polymerization and are other promising method for water treatment. Nanoparticles are integrated with a thin polymer film layer to provide extra membrane properties. In 2010/2011, based on Kurth and Lind's research, zeolite-polyamide TFN membranes for reverse osmosis are commercially available. They achieved higher salt rejection than regular reverse osmosis membranes. According to LG NanoH2O, Inc. research, TFN membranes show 
$99.4 \%$ salt rejection and two times higher water flux than polyamide membranes. Incorporation of $\mathrm{Ag}$ nanoparticles into thin film layer reduces biofouling, increases water permeability and shows antibacterial effects [3]. TFC membranes can also remove heavy metals from water, their application for water treatment is wide and surface properties can be changed and functionalized according to the requirements [4]. Nanofibrous membranes might be used for water desalination as well. Feng et al fabricated a PVDF nanofibrous membrane by electrospinning. According to their research, they obtained potable water ( $\mathrm{NaCl}$ concentration was lower than $280 \mathrm{ppm}$ ), where saline water consisted $6 \mathrm{wt} \% \mathrm{NaCl}$ [5]. The goal of the project is to obtain nanofiber membranes for water purification that can provide high fouling resistance and antimicrobial properties. To obtain antibacterial properties, nanoparticles ( $\mathrm{Ag}, \mathrm{ZnO}, \mathrm{CuO})$, which are well known for their antibacterial activities, will be attached to the membrane surface. To achieve the desired effect, functional groups must first be located on the membrane surface that are capable of binding the nanoparticles. The defluorination reaction developed by Ross et al. allows fluorine to be eliminated from the PVDF chain and replaced with more hydrophilic groups. This reaction can be performed using alkaline reagents such as $\mathrm{KOH}, \mathrm{NaOH}[6,7]$. As a result the hyydrophobicity of the membranes can increased. Groups were introduced to bind nanoparticles, in this project using the PVDF degradation reaction.

\section{EXPERIMENTAL}

The hydrophobic PVDF nanofibers were prepared before by electrospinning at the Technical University of Liberec (Czech Republic). Nanofibrous membranes were prepared by the lamination of 3 layers: nonwoven support (PET - 100 GSM), adhesive web and PVDF nanofibers. As an adhesive web used either polyurethane (PUR), polyamide (PA) or polyethersulfone (PES). As an alkaline agent potassium hydroxide and sodium hydroxide in pellet form (Penta Chemicals UNLIMITED) and diethylenetriamine (Sigma Aldrich) were used. Isopropanol and ethanol supplied by Penta Chemicals UNLIMITED were used to wash membranes and dissolve hydroxides. Deionized water was used to prepare solution and rinse membranes surfaces.

\subsection{Alkaline treatment with $\mathrm{NaOH}$ and $\mathrm{KOH}$}

Membranes were washed in isopropanol and rinsed with deionized water before modification. The membranes were then dried at $40^{\circ} \mathrm{C} .2 \mathrm{M} \mathrm{NaOH}$ solution was prepared in ethanol while $2 \mathrm{M} \mathrm{KOH}$ solution was prepared in isopropanol. The reaction was run for 0.5 and 2 hours. After treatement the membranes were washed with deionized water and dried at $40^{\circ} \mathrm{C}$. The modification was conducted at a temperature of $25^{\circ} \mathrm{C}$.

\subsection{Alkaline treatment with dietylenetriamine (DETA)}

Membranes were washed in isopropanol and rinsed with deionized water before modification. The membranes were then dried at $40{ }^{\circ} \mathrm{C}$. Membranes were modified in $4 \mathrm{M}$ aqueous DETA solution for 1,3 and 5 hours at $60{ }^{\circ} \mathrm{C}$. After treatment the membranes were washed with deionized water and dried at $40{ }^{\circ} \mathrm{C}$.

\subsection{Membrane characterization}

The scanning electron microscopy (SEM) and Fourier transfer infrared spectroscopy (FTIR) were used to estaminate changes in nanofibers structure and surface content. The Vega 3SB scanning electron microscope (SEM; TESCAN VEGA, Brno, Czech Republic) was used to observe nanofibers surface. The samples were covered with $7 \mathrm{~nm}$ gold layer before the analysis. The diameter of nanofibers was measured by ImageJ program (National Institute of Health). The samples were analyzed by Nicolet iZ10 Fourier transform infrared spectroscope (FTIR, Thermo Scientific, Prague, Czech Republic). The water contact angle was determined by Drop Shape Analyzer DS4 (Krüss $\mathrm{GmbH}$ ). Membrane pore size was determined by bubble point method using Porometer 3G through a pore size analyzer (Quantachrome Instruments, Anton Paar GmbH). 


\section{RESULTS}

In this research work it was noticed that the layers of nanofibers were separated from the membrane support when treated for long period of time or at high concentration levels of alkaline agent. The adhesive webs used did not have sufficient chemical resistance against the chemicals used resulting in damage during modification. The smallest chemical resistance was shown by PES- adhesive web membranes. The nanofibers increased their volume after the modification, independently from the chemical agent used. The diameter of pristine PVDF nanofibers was $198 \mathrm{~nm}$ while after modification it could reach up to $300 \mathrm{~nm}$. The mean pore size of pristine membranes was cca. $0.7 \mu \mathrm{m}$ whereas after alkaline treatment it became smaller and stood at 0.3-0.4 $\mu \mathrm{m}$. The differences are visible on SEM images shown in Figure 1.
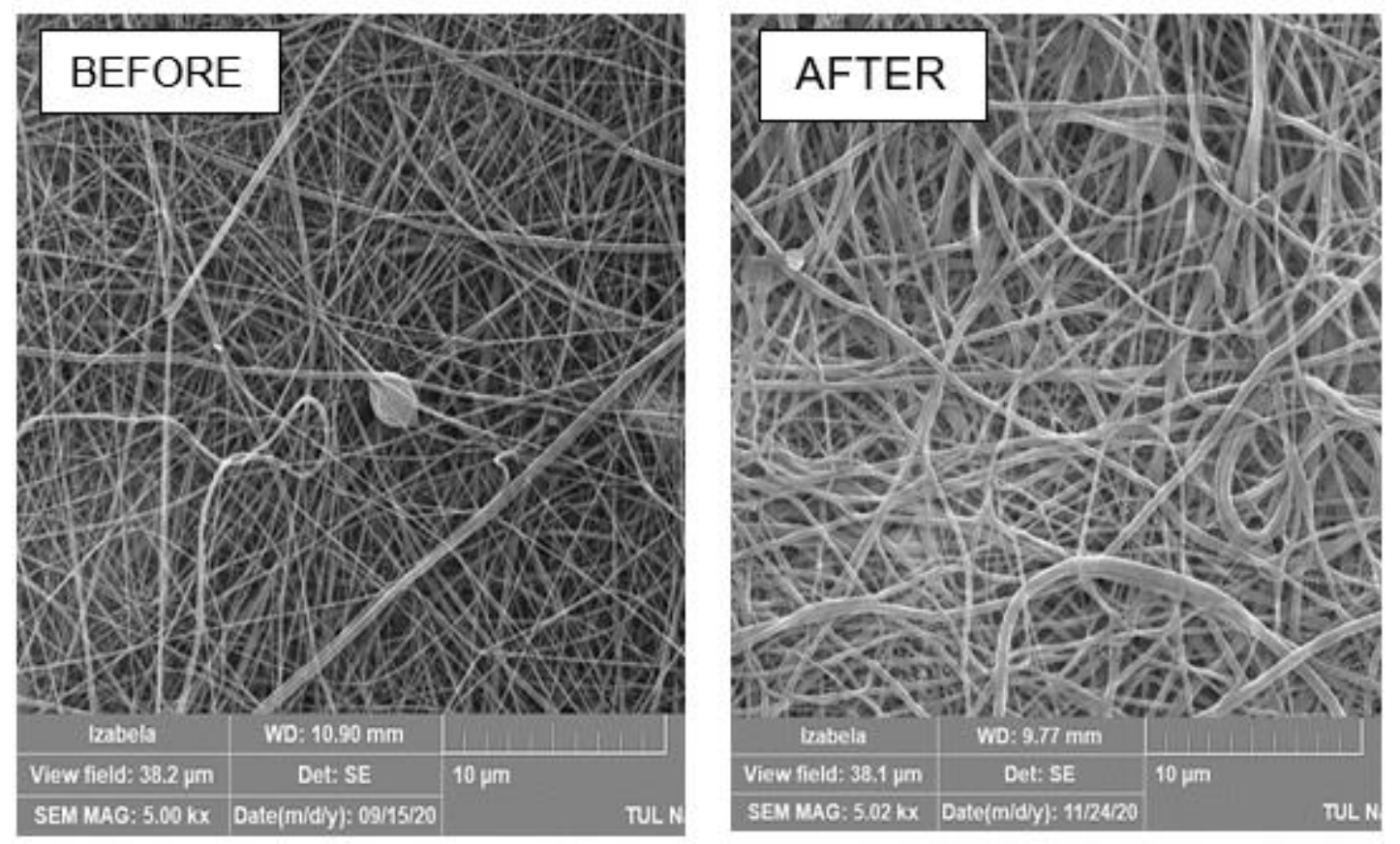

Figure 1 The PVDF nanofibers before and after modification

The measurements of contact angle show that the hydrophilicity of the modified membranes significantly increased after modification. The contact angle of pristine membranes was $97.1^{\circ}, 81.9^{\circ}, 93^{\circ}$ for PA-, PES-, PUR-based membranes, respectively. The contact angle decreased gradually with the time when treated with $\mathrm{KOH}$, whereas after others modifications reached $0^{\circ}$. Below on Figure 2 showed selected results.
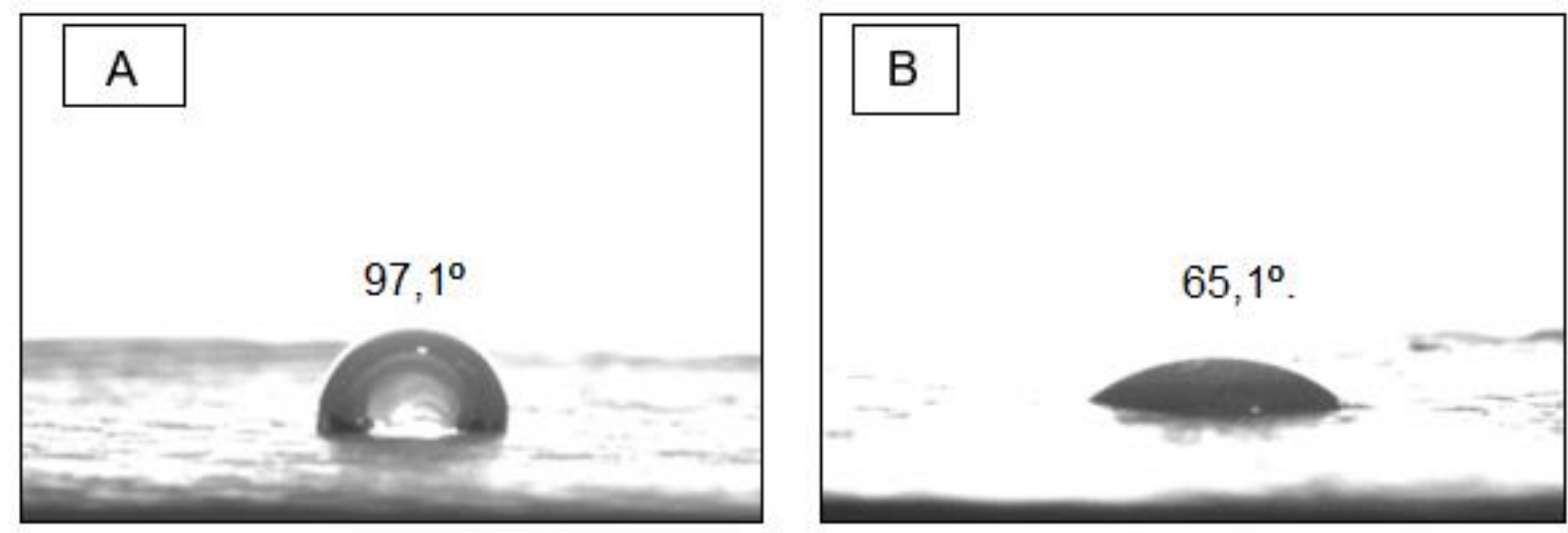

Figure 2 The contact angle for pristine PVDF membrane and after 1 hour of modification in $\mathrm{KOH}$ 
The increase in membrane hydrophilicity may indicate that some of the fluorine has been removed from the membrane and replaced with more hydrophilic groups. The FTIR results have shown changes in the membrane structure and may confirm the above assumption.

The presence of $-\mathrm{OH},-\mathrm{CF},-\mathrm{C}=\mathrm{C}$ and $\mathrm{C}=\mathrm{O}$ groups is essential to estaminate the changes in PVDF membranes sturcture. In the range of $1050-1250 \mathrm{~cm}^{-1}$ two sharp peaks appear from the vibrations of bihalide. The $-\mathrm{OH}$ groups shown near $3400 \mathrm{~cm}^{-1}$, the radical forms of $-\mathrm{OH}$ groups may occurs either in dissociation (3580-3650 $\left.\mathrm{cm}^{-1}\right)$ or in association $\left(3200-3400 \mathrm{~cm}^{-1}\right)$. In the range of $1600-1750 \mathrm{~cm}^{-1}$ peaks appear from $\mathrm{C}=\mathrm{O}$, $\mathrm{C}=\mathrm{C}$ bonds, their presence in samples may suggest that reaction of defluorization has not been completed. In the Figure 3 gives the FTIR results after $\mathrm{KOH}$ modification.
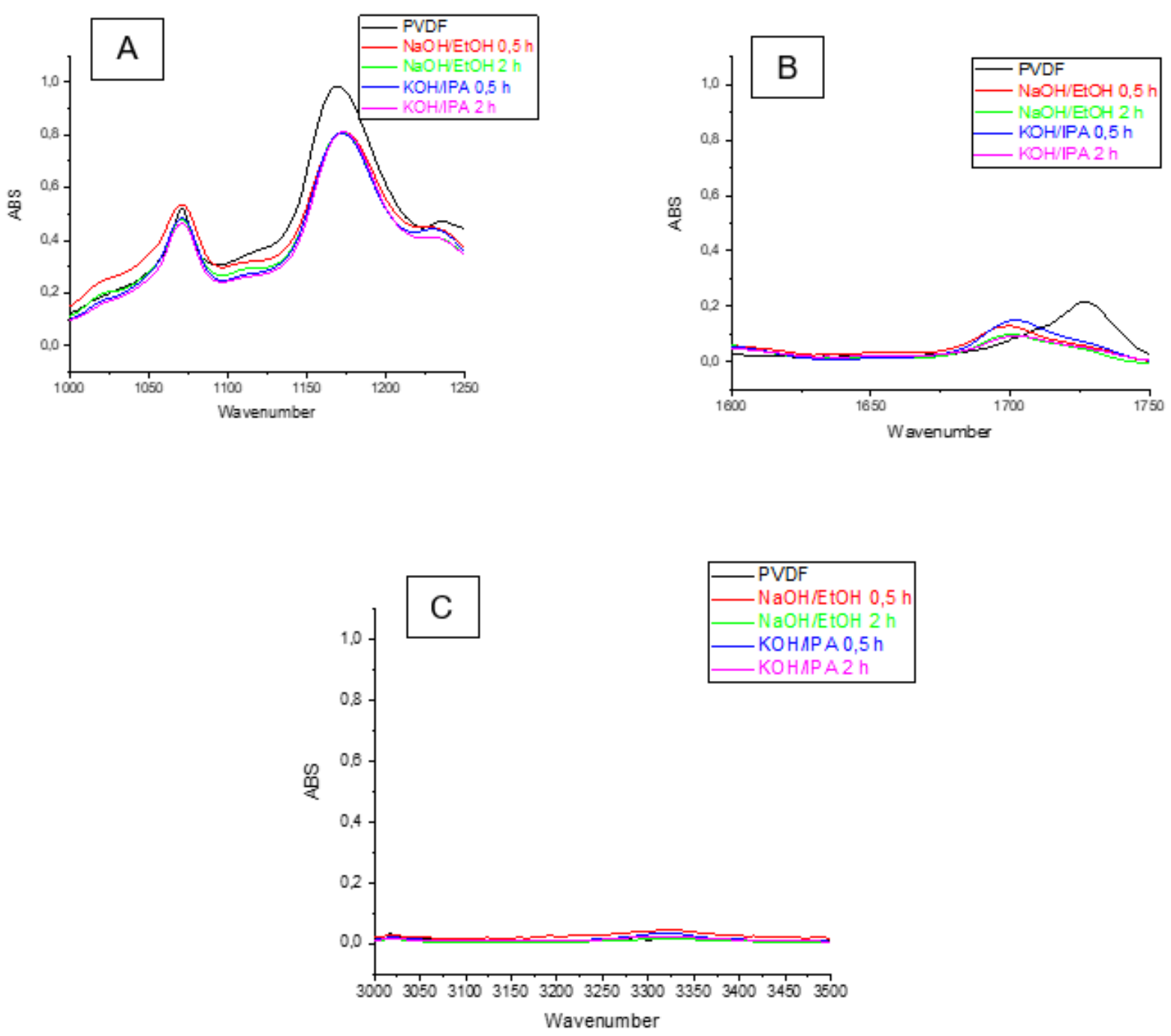

Figure 3 The FTIR results in ranges A) $1000-1250 \mathrm{~cm}-1$, B) $1600-1740 \mathrm{~cm}-1$, C) $3000-3500 \mathrm{~cm}-1$ for PVDF membranes after treatment in $2 \mathrm{M} \mathrm{KOH}$ or $2 \mathrm{M} \mathrm{NaOH}$ after 0.5 and 2 hours

The results show that the amount of $\mathrm{C}-\mathrm{F}$ groups is reduced after alkaline treatment. Determination of the presence of $-\mathrm{OH}$ groups was not possible based on the results obtained. However, the intensivity of peaks for $-\mathrm{C}=\mathrm{O},-\mathrm{C}=\mathrm{C}$ may suggest, that the reaction of defluorization was initiated, but not completed. 

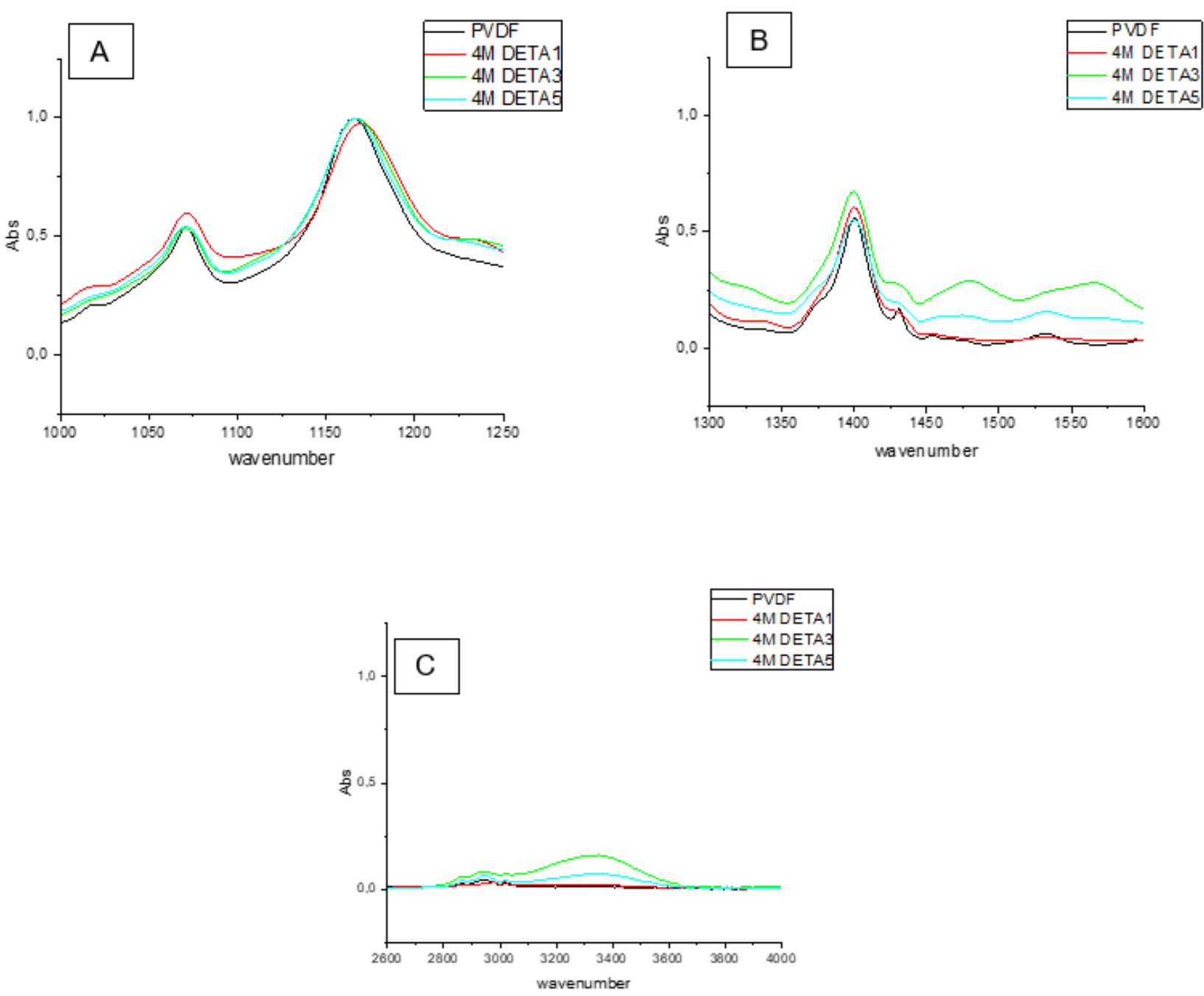

Figure 4 The FTIR results in ranges A) $1000-1250 \mathrm{~cm}-1$, B) $1300-1600 \mathrm{~cm}-1$, C) $2600-4000 \mathrm{~cm}-1$ for PVDF membranes after treatment in $4 \mathrm{M}$ DETA after 1,3 and 5 hours

It can be seen in Figure 4 that clear deformation vibrations of $\mathrm{N}-\mathrm{H}$ bonds from amine (approx. $1490+1580$ $\mathrm{cm}-1$ ) appeared after modification of membranes in 4M DETA solution. The stretching vibrations of $\mathrm{N}-\mathrm{H}$ bonds might be obscured by the stretching vibrations of $\mathrm{O}-\mathrm{H}$ bonds from residual moisture. However, it can be concluded that $-\mathrm{NH}_{2}$ groups appeared on the membrane surface.

\section{CONCLUSION}

According to the results obtained, alkaline treatment by $\mathrm{KOH}$ and $\mathrm{NaOH}$ does not provide sufficient amount of $-\mathrm{OH}$ groups into surface of nano PVDF membranes. The high concentration of alkaline agent causes membrane disintegration and leads to disassociation of layers. The small concentration of modified agents initiates a reaction of defluorization, but is not completed. However, in the literature, where fluoride was successfully removed from the polymer chain and replaced by -OH groups. Reducing the concentration of the alkaline agent and increasing the reaction time may be the key to success. Modification with DETA yielded $\mathrm{NH} 2$ groups on the membrane surface that could be used to attach nanoparticles in the future. 


\section{ACKNOWLEDGEMENTS}

The research presented in this article was partly supported by the Internal Grant of the Technical University of Liberec, Czech Republic (Project No. SGS-2021-3027). The authors acknowledge the assistance provided by the Research Infrastructures NanoEnviCz (Project No. LM2018124), which is supported by the Ministry of Education, Youth and Sports of the Czech Republic.

\section{REFERENCES}

[1] SINGH, R., BHADOURIA, R., et al. Nanofiltration technology for removal of pathogens present in drinking water. Waterborne Pathogens. 2020, p.463-489.

[2] PATANJALI, Pooja, et al. Nanotechnology for water treatment: A green approach. In: Green Synthesis, Characterization and Applications of Nanoparticles. Elsevier. 2019, p. 485-512.

[3] REDDY K., et al. Nanotechnology for water purification: applications of nanotechnology methods in wastewater treatment. In: Water Purification. Academic Press. 2017, p. 33-74.

[4] MADHURA, Lavanya; SINGH, Shalini. A Review on the Advancements of Nanomembranes for Water Treatment. Nanotechnology in Environmental Science. 2018, pp. 391-412.

[5] WANG, Zhuqing, et al. Recent advances in nanoporous membranes for water purification. Nanomaterials. 2018, vol. 8, no. 2, p. 65.

[6] ROSS, G. J., et al. Surface modification of poly (vinylidene fluoride) by alkaline treatment1. The degradation mechanism. Polymer. 2000, vol. 41, no. 5, pp. 1685-1696.

[7] ROSS, G. J., et al. Surface modification of poly (vinylidene fluoride) by alkaline treatment Part 2. Process modification by the use of phase transfer catalysts. Polymer. 2001, vol. 42, no. 2, pp. 403-413. 